Ebisu Ebisu

Études japonaises Études japonaises

48 automne-hiver 2012

Naissance d'une revue féministe au Japon : Seitō

(1911-1916)

\title{
Le débat dans Seitō sur la femme nouvelle, atarashii
} onna

The Debate in Seitō on New Women, Atarashii Onna

『青鞮』における「新しい女」に関する論争

\section{Marion Saucier}

\section{OpenEdition}

Journals

Édition électronique

URL : http://journals.openedition.org/ebisu/605

DOI : 10.4000/ebisu.605

ISSN : 2189-1893

Éditeur :

Institut français de recherche sur le Japon (UMIFRE 19 MAEE-CNRS), Maison franco-japonaise

Édition imprimée

Date de publication : 1 septembre 2012

Pagination : 83-99

ISSN : 1340-3656

Référence électronique

Marion Saucier, «Le débat dans Seitō sur la femme nouvelle, atarashii onna », Ebisu [En ligne], 48 | automne-hiver 2012, mis en ligne le 03 avril 2014, consulté le 02 mai 2019. URL : http:// journals.openedition.org/ebisu/605; DOI : 10.4000/ebisu.605 


\title{
Le débat dans Seitō sur la femme nouvelle, atarashii onna
}

\author{
Marion SAUCIER*
}

L'expression "New Women » a circulé aux États-Unis et en Europe à la fin du XIX ${ }^{\mathrm{e}}$ siècle, et elle devient à la mode au Japon après des conférences de Tsubouchi Shōyō 坪内覓遥 ${ }^{1}$ en juillet 1910 au sujet des héroïnes de théâtre occidentales ${ }^{2}$. Elle a d'abord été utilisée par les journalistes pour parler des membres de la revue Seitō 青靱 sur un ton curieux et ironique, mais plutôt bienveillant. En mai 1912, le journal Yomiuri shinbun 読売新聞 publie une série d'articles intitulée Atarashii onna 新しい女 (Les femmes nouvelles). Or, après la visite à Yoshiwara 吉原 ${ }^{3}$, les membres du groupe sont très critiquées, et l'équipe elle-même connaît des débuts de dissensions. Lexpression atarashii onna est devenue un anathème. Hiratsuka Raichō 平塚らいてう (1886-1971) s'en saisit et rédige un article pour la revue Chūō kōron 中央公論, puis elle propose de réaliser un numéro de Seitō autour de cette question : il en résultera les numéros 1 et 2 du volume 3 , en janvier et février 1913. On y trouve les signatures de Hiratsuka Raichō, fondatrice du groupe, et d'Itō Noe 伊藤野枝 (1895-1923), qui dirigera la revue à partir

* Centre d'études japonaises, INALCO.

1. Tsubouchi Shōyō (1859-1935), écrivain, dramaturge et critique littéraire, qui enseignait à l'université Waseda.

2. Voir à ce sujet l'article consacré dans ce dossier au débat sur La Maison de poupée et le personnage de Nora d'Ibsen.

3. En juillet 1912, trois membres de Seitōo, dont Hiratsuka Raichō, effectuent une visite à Yoshiwara, le quartier des courtisanes de Tokyo, ce qui provoque un scandale. 
de 1915, ainsi que d'Iwano Kiyo 岩野清 (1882-1920) et de Katō Midori 加藤緑 (1888-1922) qui y contribuent régulièrement, et, à titre exceptionnel, les noms de Fukuda Hideko 福田英子 (1865-1927), féministe de la génération précédente, sollicitée pour apporter son poids moral au numéro, ou encore de Hori Yasu 堀保, épouse du célèbre anarchiste Ōsugi Sakae 大杉栄 (1885-1923), qui signe un texte paradoxal intitulé « Watashi wa furui onna desu» 私は古い女です(Je suis une femme ancienne).

La série d'articles du Yomiuri et le numéro spécial de Chūō kōron sur la femme nouvelle montrent que la question est dans l'air et que Seitō n'est pas la seule revue à s'intéresser au sujet. Le numéro de Chūō köron comprend un texte sur le droit de vote des femmes, un sur l'homosexualité, un sur la vie quotidienne des veuves ou encore sur la comparaison des femmes occidentales et des femmes japonaises. Les femmes commencent à prendre leur place dans l'espace public, la révolution industrielle a rendu leur maind'œuvre indispensable dans le secteur du textile par exemple, et dans la société se font jour des pressions pour une reconnaissance de leurs droits politiques, à travers notamment la remise en cause de l'article 5 de la loi sur l'ordre public et la police qui interdit aux femmes depuis 1890 de participer à tout rassemblement politique.

Mais, première revue de femmes, Seitō offre l'originalité d'un regard pluriel par des femmes, sur elles-mêmes. Et de fait, les articles qui nous intéressent ici sont très différents les uns des autres. Nous présentons donc ci-dessous trois manières ou styles de traitement du thème de la femme nouvelle. Hiratsuka Raichō et Itō Noe lancent des appels vibrants et parfois exaltés aux femmes. De leur côté, Iwano Kiyo et Katō Midori proposent des arguments rationnels, des raisonnements pour convaincre. Fukuda Hideko, enfin, offre une solution globale à la question de la femme. Nous verrons aussi l'élan commun qui anime les auteurs et nous mettrons en lumière, en guise de conclusion, l'actualité du propos contenu dans ces textes ${ }^{4}$.

4. Nous nous appuierons sur le recueil d'articles de Seitō publié en 1991 par Horiba Kiyoko, à l'exception du texte de Hori Yasu, pour lequel nous avons consulté l'édition de Seitō en fac-similé publié par Fuji shuppan 不二出版 en 1983. 


\section{La mission des femmes nouvelles}

Hiratsuka Raichō dans « Ren.ai to kekkon : Eren Kei no cho » 恋愛と結婚 —エレン•ケイの著 (Amour et mariage : l'ouvrage d'Ellen Key) et Itō Noe dans le texte "Atarashiki onna no michi » 新しき女の道 (La voie des nouvelles femmes) ont en commun d'appeler les femmes à s'engager sur une voie difficile. Comme le laisse entendre la notion de "voie ", le ton de leur propos est presque religieux par moment. Pour Raichō, les nouvelles femmes ont une vocation. Pour Itō Noe, elles ont leur propre voie, qu'elles doivent ouvrir elles-mêmes.

Ce chemin est semé d'embûches, cette voie est un combat. Raichō parle d'efforts et de tourments (on pense au combat sans merci évoqué par Nora dans La Maison de poupée d'Ibsen). Itō Noe fait une description effrayante du sort qui attend les pionnières :

"Sur la voie encore inconnue que doivent emprunter les pionnières, il leur faut se frayer un chemin entre les ronces pleines d'épines qui entravent leur route. Elles doivent abattre d'énormes rochers pour avancer et se perdre dans des montagnes profondes. Piquées par des insectes venimeux, souffrant de la faim et de la soif, elles doivent franchir des cols, escalader des falaises et traverser des vallées en s'agrippant aux racines des plantes. Ce faisant elles doivent contenir leurs hurlements, leurs prières et leurs larmes amères devant tant de souffrances. " (Horiba 1991 : 93).

Pour Itō Noe, ces pionnières sont une élite, une avant-garde qui conduit les femmes, et qui seule mérite le qualificatif de « nouvelle».

« Les pionnières ne permettent en aucun cas que l'on se mêle de leur travail. Elles ne s'intéressent pas non plus à celles qui les suivent. Celles qui les suivent n'ont aucun statut pour les critiquer en quoi que ce soit. Elles n'ont aucun droit. Elles n'ont qu'à marcher sur les traces des pionnières avec gratitude. Elles ne savent pas avancer par elles-mêmes. Elles ne peuvent guère qu'avancer sur les traces des pionnières en se servant de leur exemple. » (Horiba 1991 : 94).

Les hommes sont quasiment absents de ce tableau, il s'agit d'une affaire strictement féminine et d'une émulation entre femmes.

Si Itō Noe est presque effrayante par sa virulence, sans doute explicable par son très jeune âge (elle est née en 1895), et par sa description du bataillon des femmes pionnières, Raichō mêle habilement des passages résolument positifs : "Je suis une femme nouvelle " (Horiba 1991 : 90) à 
des phrases plus impératives : "Les femmes nouvelles ne supportent pas de marcher sur les traces des anciennes " (Horiba 1991 : 91), " les femmes nouvelles ne se satisfont pas de la vie des femmes d'avant, abêties, réduites en esclavage et corrompues à cause de l'égoïsme des hommes " (Horiba 1991 : 91), exprimant des vœux pour le futur : "Les femmes nouvelles souhaitent détruire l'ancienne morale et les vieilles lois élaborées pour la commodité des hommes» (Horiba 1991 : 91), mais citant les obstacles : "Mais les fantômes qui se sont emparés de la tête des femmes d'autrefois poursuivent avec acharnement les femmes nouvelles " (Horiba 1991 : 91), pour aussitôt proposer de « construire un nouveau royaume, avec une nouvelle religion, une nouvelle morale, de nouvelles lois" (Horiba 1991 : 91).

Itō Noe et Raichō mobilisent clairement les femmes, elles ont un discours dynamique (même si Raichō se défend de vouloir créer un mouvement), et elles ont aussi en commun de ne jamais vraiment définir ces femmes nouvelles concrètement. Elles veulent détruire la morale, la religion, les lois existantes, mais on n'a pas idée de ce qui est proposé à la place. Itō dit bien qu'elle ne sait pas d'où vient cette voie ni où elle mène. Raichō ne nous dit pas non plus clairement ce qu'elle entend par "femme nouvelle ", et elle pose même ouvertement la question : "Alors ce royaume qu'en est-il ? Cette nouvelle religion, qu'est-ce ? Les femmes nouvelles ne le savent pas encore. Mais elles étudient, se forment, multiplient les efforts et souffrent pour ce qu'elles ne connaissent pas encore " (Horiba 1991 : 92).

À l'opposé de ce langage de combat, les textes d'Iwano Kiyo et de Katō Midori adoptent un ton plus posé et proposent une réflexion basée sur des arguments rationnels.

\section{Des femmes nouvelles inscrites dans I'histoire}

Dans le texte intitulé " Jinrui to shite dansei to josei wa byōdō de aru » 人類 として男性と女性は平等である (En tant qu'êtres humains, les hommes et les femmes sont égaux), Iwano Kiyo ${ }^{5}$ compare les hommes et les femmes, et

5. Iwano Kiyo fut d'abord institutrice avant de devenir journaliste. Elle se mobilisa contre l'article 5 de la loi de police sur l'ordre public, qui empêchait les femmes de participer à des regroupements politiques. 


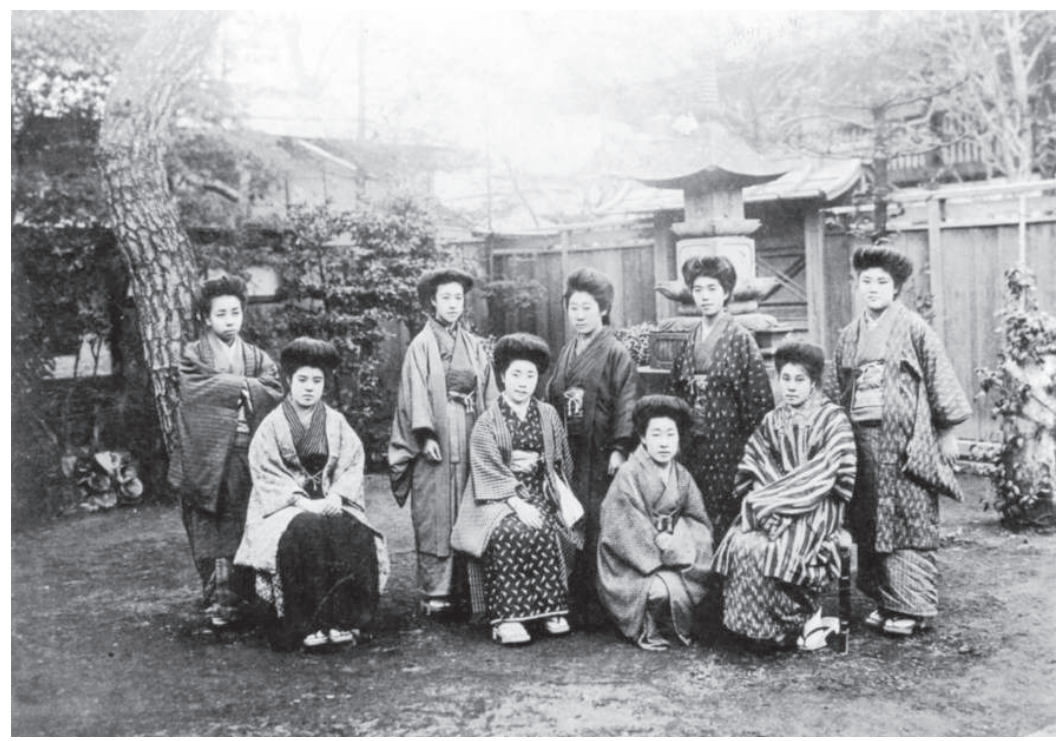

Fig. 1. Photo des membres de Tokyo de la Seitōsha, prise le 11 décembre 1911, après une réunion dans le jardin du temple Shōrin-ji 勝林寺 à Mannen yama 万年山 (Tokyo, Hongō) publié dans le numéro de janvier 1912, p. 170. Hiratsuka Raichō, deuxième personne debout à partir de la gauche.

expose un raisonnement logique pour montrer combien l'idée de la supériorité des hommes sur les femmes est stupide. Elle explique que, par exemple, on entend souvent dire qu'un homme vaut deux femmes par sa force et donc qu'il leur est supérieur : dans ce cas les lutteurs de sumo doivent être supérieurs aux autres hommes, ou les bêtes sauvages telles que les ours ou les tigres doivent être supérieures aux hommes. Un autre argument souvent évoqué est celui de la physiologie et des menstruations, ainsi que les grossesses des femmes, qui sont censées les affaiblir et les rendre moins productives. Iwano Kiyo montre que les grossesses par exemple ne concernent que les femmes mariées et n'ont lieu au plus qu'une année sur deux ou trois, donc ne justifient pas qu'on les invoque pour dénigrer la productivité des femmes. Comme le souligne Vera Mackie (Mackie 2003 : 48-49), cette remise en cause des justifications physiologiques de la discrimination envers les femmes est très dérangeante pour la société de l'époque. 
L'argument d'Iwano Kiyo le plus intéressant est cependant celui qui porte sur la connaissance. D'emblée, au début du texte, elle reconnaît une supériorité provisoire aux hommes dans ce domaine. Mais elle compare les relations entre hommes et femmes aux relations entre le Japon et les pays occidentaux. Au début de l'ère Meiji, le Japon était en retard sur les pays d'Europe ou les États-Unis. Il a rattrapé une partie de son retard. Était-il juste que les Occidentaux traitent les Japonais en inférieurs ? De la même façon, les femmes maintenues dans l'ignorance ont un niveau de connaissance bien inférieur à celui des hommes, mais ceux-ci n'ont qu'une simple avance (Iwano Kiyo les qualifie de senpai 先輩, d' "aînés "), lorsque les femmes auront eu droit à l'éducation comme les hommes, elles les rattraperont. Est-il juste que les hommes les considèrent comme des inférieures?

Iwano Kiyo s'appuie sur les principes d'égalité, de liberté - et plus précisément la liberté de pensée - et de droit. Ainsi, quand elle évoque les caractéristiques physiologiques de la femme, elle affirme :

"À supposer que du fait de nombreuses grossesses, une gêne soit occasionnée, cela ne justifie pas que l'on restreigne l'égalité du droit à la liberté de pensée que l'être humain civilisé doit se voir attribuer sans distinction. » (Horiba 1991 : 98).

Par rapport aux autres textes, celui d'Iwano Kiyo se distingue aussi par le fait qu'il évoque les activités réelles des femmes, leur implication déjà effective dans la société, ce qui donne un éclairage très différent de la vision noire d'Itō Noe par exemple.

« [...], le cycle menstruel des femmes est un processus naturel. Ce n'est absolument pas une maladie. Pour une femme en bonne santé, ce n'est pas une douleur suffisante pour lui faire abandonner ses tâches ou sa charge. En témoignent dans la réalité les nombreuses femmes de notre pays qui exercent un métier et une activité. » (Horiba 1991 : 98).

Pour Iwano Kiyo, les femmes sont en permanence culpabilisées et diabolisées. Elle propose de remplacer le proverbe « Derrière tout crime il y a une femme " par le suivant : " ... derrière toute bonne action il y a une femme » (Horiba 1991 : 99). Cette idée de la culpabilisation des femmes dans la morale confucianiste notamment a déjà été mise en lumière par Fukuzawa Yukichi 福沢諭吉 dans Nihon fujin-ron 日本婦人論 (Sur les femmes japonaises) et Onna daigaku hyōron 女大学評論 (Critique de La Grande Étude des femmes) (Fukuzawa 2003). 


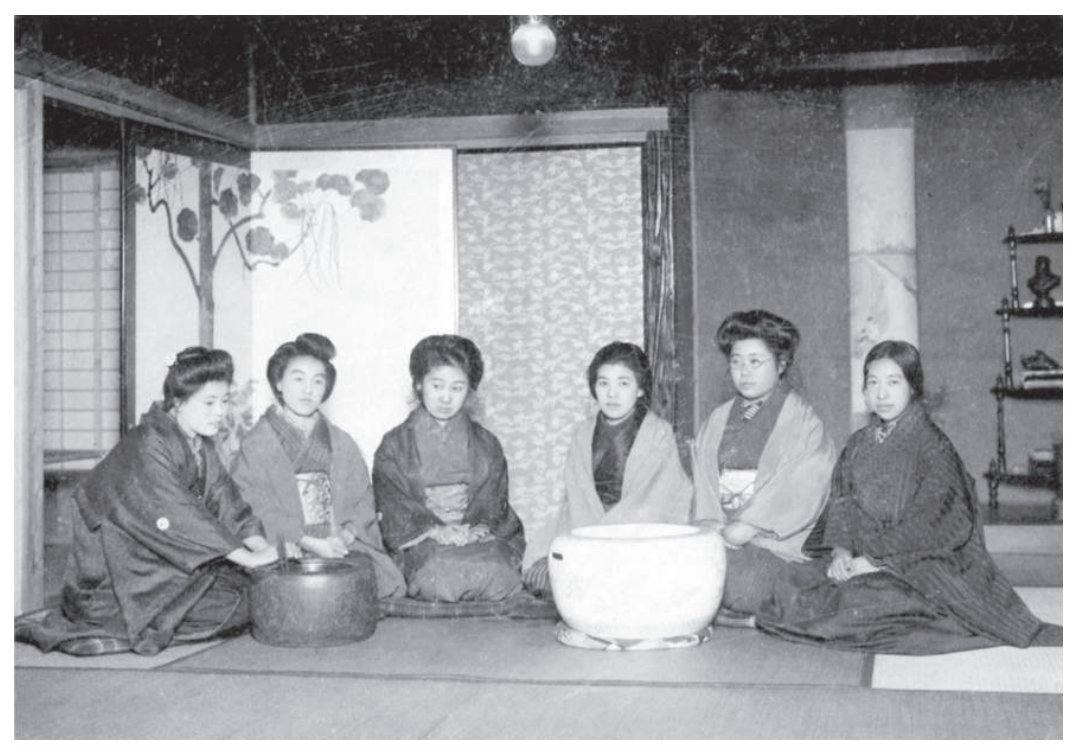

Fig. 2. Fête du nouvel an chez Iwano Kiyo. De droite à gauche : Hiratsuka Raichō, Yasumochi Yoshi 保持研 (Hakuu 白雨), Araki Iku 荒木郁, Nakano Hatsu 中野初, Iwano Kiyo, Kobayashi Katsu 小林哥津. Photo publiée dans Seitō en février 1914.

Katō Midori ${ }^{6}$, de son côté, dans " "Atarashii onna” ni tsuite "「新しい女」 について (À propos des "femmes nouvelles ») partage avec Iwano Kiyo une vision historique de la question des femmes, ainsi qu'une prise en compte du genre masculin dans son raisonnement.

"Fondamentalement, je pense que l'expression "femme nouvelle" ne désigne pas seulement des femmes nées récemment. Le sens évolue, mais il est probable qu’à chaque époque sont apparues des femmes qui ont brisé le modèle de leur temps, en vigueur jusque-là. » (Horiba $1991: 101)$.

Par ailleurs, pour l'époque qui est la sienne, Katō Midori fait remonter la nouveauté à la génération précédente et à Fukuda Hideko : "Quoi qu’il

6. Katō Midori a publié plusieurs nouvelles dans la revue. Sa vie fut un combat pour concilier son désir de s'exprimer comme écrivain et ses obligations de mère de famille. 
en soit, les femmes nouvelles ne datent pas d'aujourd'hui ; en 1888-1889, il y a déjà eu Fukuda Hideko qui, toute femme qu'elle était, s'est levée pour réclamer la liberté au moment où le pays changeait de politique. " (Horiba 1991 : 102). Elle a conscience de son époque comme d'une phase de transition et se projette cinquante ou cent ans plus tard (c'est-à-dire exactement à notre époque) :

"Surtout que l'état de la société contemporaine n'est pas encore entré dans une époque nouvelle. Prisonnières de ce choc entre le neuf et l'ancien, les femmes ne savent pas où aller. Peut-être que les femmes dans cinquante ou cent ans baigneront dans la lumière du bonheur, mais nous, nous devrons probablement passer notre vie avec ces doutes et ces tourments. " (Horiba 1991 : 106).

S’interrogeant sur les éléments qui déclenchent l'apparition des femmes nouvelles, elle remarque que c'est lors de périodes troublées, quand les hommes rencontrent des difficultés, que les femmes relèvent la tête :

«Comme la crise de l'État met en valeur les ministres fidèles, comme l'appauvrissement de la famille révèle le fils pieux, notre époque de transition où religion et morale sont perturbées et confuses, a donné soudain naissance à cette "femme nouvelle".

"Jusqu'ici la tête rabaissée par l'homme, entravée par les trois autorités" auxquelles elle devait se soumettre, la femme qui n’avait même pas le droit de réfléchir à l'écriture, au monde extérieur et encore moins évidemment à sa propre image, a tout à coup relevé la tête et rejeté la lourde main de l'oppression. De plus, c'est parce que cette main a hésité que les "femmes nouvelles" ont relevé la tête. En effet, jusqu’à maintenant les hommes utilisaient les femmes comme des instruments. Tout se faisait selon la volonté des hommes. En même temps, ces derniers portaient l'entière responsabilité de la vie quotidienne. On considérait qu'entretenir la femme constituait la tâche normale de l'homme, mais bien sûr les hommes actuels sont assaillis par les difficultés et les tourments mondiaux de notre époque, et par le doute, et ils se trouvent maintenant dans une position extrêmement pénible. En conséquence, ils ne peuvent s'échapper des difficultés de la vie courante qui envahissent leur quotidien, ni des affres et des tourments de la pensée. Soudain l'individualisme ne leur laisse plus le loisir d'entretenir les femmes. Aussi, en même temps que les droits des hommes sur les femmes diminuaient, les droits des femmes augmentaient. » (Horiba 1991 : 102-103).

7. Autorité du père, puis du mari, puis du fils. 
D'autre part, des circonstances contingentes expliquent aussi cette émergence des femmes nouvelles. Comme dans les pièces d'Ibsen ou de Sudermann, des événements, des circonstances peuvent amener la femme à l'éveil, à la prise de conscience. Les femmes veulent alors observer : " Elles assistent froidement à leur destin qui s'avance. Elles observent, immobiles, d'un regard critique, les événements de la société. N'est-ce pas là l'attitude des femmes nouvelles ? (Horiba 1991 : 104). "Froidement ", " immobiles " : on est très loin de la longue marche et des souffrances des pionnières d'Itō Noe.

Katō Midori analyse ensuite le chemin que doivent suivre les femmes nouvelles, et son regard se fait critique sur les «femmes nouvelles " de son époque, celles qui gravitent autour de Seitō :

«Premièrement, elles ne doivent pas dépendre des hommes; elles doivent être indépendantes en ayant un métier [...] En tout cas, à partir du moment où il ne faut pas dépendre des hommes, il faut sans doute être célibataire. Apparemment, nombreuses sont les femmes d'aujourd'hui qualifiées de nouvelles qui sont célibataires. De plus, il semble que beaucoup d'entre elles ne connaissent pas encore le goût amer de la vie. Quoi qu'on en dise, je pense que les choses ne sont pas sérieuses tant qu'on vit auprès de ses parents, et qu’on vit à sa guise avec leur argent. " (Horiba 1991 : 105).

Katō Midori semble ici mettre en cause le sérieux des jeunes femmes qui n'ont pas encore fait l'expérience de la vraie vie, dont sans doute une partie des membres et des sympathisantes de Seitō.

Indépendance financière et célibat sont nécessaires pour être une "femme nouvelle » et Katō Midori remarque qu'à son époque, les seules qui s'en sortent facilement sont des artistes de tous domaines, et que cette caractéristique attire le regard de l'opinion publique.

Elle se demande alors si le célibat est possible à long terme et conclut par la négative, ce qui pose la question du type de mariage que ces femmes peuvent espérer : pour Katō Midori, même si elles ont la chance de tomber sur un "homme nouveau», elles devront renoncer à une partie d'ellesmêmes, à plus forte raison si elles ont des enfants :

« S'il faut absolument se tenir debout sous le nom d'épouse ou de mère, plutôt que de se tenir en tant qu'être humain, alors il faudra entendre le triste anathème "ton nom est femme". À supposer que l'on ne dépende pas de son mari, c'est-à-dire que les deux réussissent à conduire leurs activités et que l'on puisse diviser les droits 
par deux, ne serons-nous pas face à une contradiction, un choc inexprimable ? En somme, homme et femme au bout du compte ne peuvent se comprendre. Le monde des hommes et le monde des femmes sont complètement différents. " (Horiba 1991 : 106).

Il est intéressant de constater que Katō Midori envisage le rôle des hommes nouveaux, mais que sa vision pessimiste du mariage ne s'en trouve pas modifiée. Les perspectives qu'elle trace pour les jeunes femmes de son époque sont sombres, même si cela est exprimé de façon beaucoup plus sereine que chez Itō Noe.

\section{Une nouvelle société pour une femme nouvelle}

Fukuda Hideko est atypique dans ce débat. Socialiste, elle n'est pas membre de Seitō. Son texte est intitulé "Fujin mondai no kaiketsu » 婦人問題の 解決 (La solution au problème des femmes). Dans son autobiographie, Raichō raconte l'avoir sollicitée pour avoir le point de vue d'une socialiste (ce qui vaudra l'interdiction de vente de ce numéro de Seitō). Elle dit avoir été impressionnée par la rigueur du propos de Fukuda, qui écrivait " comme un homme" (Hiratsuka 1992, vol. 2 : 89-103). La caractéristique de Fukuda est donc de proposer ici une solution globale à la question des femmes : c'est l'avènement du communisme qui est censé résoudre tous les problèmes des femmes. Elle avance son raisonnement en trois points :

1. Qu'entend-on par libération ? Il ne s'agit pas uniquement de l'obtention des mêmes droits que les hommes, comme elle l'avait cru au moment du Mouvement pour la liberté et les droits du peuple, ce qui ne constituerait qu'une libération relative, comme le mouvement pour les droits politiques des femmes ou le mouvement pour l'accès des femmes à l'enseignement supérieur. Il faut viser la libération absolue, c'est-à-dire une " libération en tant que personne et non en tant que femme simplement. Il s'agit d'assurer la liberté de la personne et non la liberté de la femme » (Horiba 1991 : 110).

2. Raichō dit qu'elle est le soleil ${ }^{8}$, mais en fait tout être humain devrait avoir cette conviction : " (...) ce n'est pas seulement le problème des

8. Dans le premier numéro de Seitō, Raichō avait publié : «À l'origine, la femme était le soleil " (voir l'introduction au dossier dans ce numéro) [N.D.L.R.]. 
femmes, mais il faut aussi que les hommes puissent avoir cette conscience. Il faut que la libération des hommes intervienne en même temps que celle des femmes" (Horiba 1991 : 110). Fukuda souligne la difficulté du sort des hommes à son époque et idéalise la société primitive, censée avoir assuré la liberté et une vie saine aux hommes comme aux femmes ${ }^{9}$. C'est la civilisation qui les a séparés et qui a créé les classes sociales. Une société sans science, sans technique et sans machine était une société pacifique et joyeuse où régnait le bonheur.

3. C'est le communisme qui permettra de libérer les êtres humains de cette situation, aussi bien les hommes que les femmes : "À l'aube du communisme, amour et mariage deviendront naturellement libres " (Horiba 1991 : 111). Elle réfute par avance l'idée que cette liberté pourrait s'accompagner de débauche ou de destruction de la famille : dans une société débarrassée de la propriété privée, plus de mariage pour l'argent, et seul l'amour véritable devient le lien unissant les époux. Plus de nécessité de détruire la famille (qui d'ailleurs, dit-elle, ne se laisserait pas détruire si facilement). La science et la technique seront au service de tous et la femme ne sera plus exploitée dans le foyer, elle aura plus de temps et de force pour elle-même. Sans cette transformation radicale de la société, l'obtention des droits politiques ne sera valable que pour une partie des femmes et la lutte des classes qui sévit entre les hommes s'étendra aux femmes.

Ainsi, la position de Fukuda Hideko, sans surprise, est très particulière dans ce débat et peut difficilement se comparer aux autres.

Une mention particulière doit être faite du texte de Hori Yasu, "Je suis une femme ancienne ». Ce texte n'a pas été repris dans l'édition de poche regroupant les textes les plus marquants de Seitō. À première vue, il est de peu d'intérêt pour notre sujet, puisque justement son auteure, qui partage la vie d'Ōsugi Sakae ${ }^{10}$, se proclame étrangère à ce combat des femmes nouvelles. Mais l'éclairage qu'elle apporte sur la réalité des femmes de son

9. On peut lire ici l'influence de F. Engels, L'Origine de la famille, de la propriété privée et de l'État, traduit de l'anglais par Sakai Toshihiko 堺利彦 en 1907, sous le titre Danjo kankei no shinka 男女関係の進化 (L'évolution des relations entre les hommes et les femmes).

10. Anarchiste très connu, il fut assassiné avec Itō Noe et son neveu Tachibana Sōichi dans une gendarmerie dirigée par le lieutenant Amakasu au lendemain du tremblement de terre de 1923 (Amakasu jiken 甘粕事件, l'affaire Amakasu). À l'époque Ōsugi avait 
époque nous semble tout à fait intéressant, comme nous semble digne d'intérêt que la revue lui ait offert une tribune pour défendre un point de vue si différent de la ligne générale. Ayant uni sa vie à celle d'un anarchiste, Hori Yasu doit accepter de ne pas porter le nom de son compagnon pour satisfaire les idées d'Ōsugi Sakae, qui ne croit pas à l'institution du mariage et prône la liberté dans le couple. Hori Yasu exprime clairement son inquiétude face à cette liberté qu'il lui accorde, mais dont elle ne peut profiter. Elle se sent vulnérable, car elle a été élevée dans l'idée que la femme dépend de l'homme, elle a reçu très peu d'instruction et compte sur l'institution du mariage pour la protéger. Elle est donc la représentante de ces femmes à la pensée archaïque, habitées par des "fantômes ", dont parlait Raichō. Mais de fait, dans une société où les femmes ne se voient pas reconnaître l'indépendance économique, l'exposition brutale à la solitude et à l'indépendance peut avoir des effets désastreux. Le texte de Hori Yasu ne manque pas par ailleurs d'ironie, puisqu'elle reproche aux "femmes nouvelles " de la ranger d'emblée dans la même catégorie que son compagnon, de l'imaginer socialiste alors qu'elle ne partage pas les idées d'Ōsugi ${ }^{11}$. Autrement dit, les responsables de Seitō ne lui reconnaissent pas la liberté de pensée qu'elles réclament pour elles-mêmes (elle remarque d'ailleurs qu'en cela elles se comportent comme les autorités qui la font suivre parfois comme Ósugi). On peut cependant remarquer que la revue lui offre une tribune pour exprimer ouvertement son désaccord, ce qui est un indice de la grande liberté de débat qui existait dans le groupe.

\section{Les points communs de ces textes: pessimisme et défense de l'être humain}

Au-delà des différences de style et de méthode, ces quelques textes nous semblent cependant se rapprocher par un certain nombre de points.

commencé la publication de la revue Kindai shiso 近代思想 qui n’aborde pas directement les questions politiques pour éviter la censure [N.D.E.].

11. Ōsugi est connu avant tout comme anarchiste, mais à l'époque la distinction entre les deux n'était pas aussi importante qu'après les polémiques des années 1920. Yasu le qualifie de socialiste dans son texte [N.D.E.]. 
Tout d'abord, si l'on excepte Fukuda Hideko qui manifeste une grande confiance quant à la possibilité de voir arriver la société de ses rêves, les auteures sont pessimistes. Elles nous parlent de combat éternel (Itō Noe, Hiratsuka Raichō), ou en tout cas admettent qu'elles ne verront pas leur vœu de femme nouvelle s'exaucer de leur vivant (Katō Midori). Même si le tableau n'est pas toujours aussi sombre que celui d'Itō Noe, les termes de lutte, de tourment, de souffrance reviennent souvent sous leur plume. Lorsque Katō Midori envisage le célibat comme condition de l'indépendance des femmes, elle conclut à l'impossibilité de rester dans cet état toute une vie. Mais en même temps l'accent est mis sur la force de ces femmes : c'est vrai chez les pionnières d'Itō Noe, mais c'est aussi vrai pour les femmes de Katō Midori, capables d'encaisser le plus dur.

Par ailleurs, nous n'avons pas de grande précision sur ce que sera la femme demain. Là aussi Fukuda Hideko se démarque naturellement puisque la société à laquelle elle aspire est un cadre déjà bien tracé. Mais pour les autres, la question du sort de la femme et de la nature de la femme nouvelle reste ouverte (explicitement chez Raichō par exemple, pour qui les femmes se battent pour quelque chose qu' elles ne connaissent pas), et même si Katō Midori de son côté envisage une femme célibataire, la conclusion négative à laquelle elle arrive nous ramène à la question de départ : que sera la femme nouvelle?

On note ensuite l'importance de l'épanouissement personnel, notamment à travers les sentiments, chez les différentes auteures. Fukuda dit bien qu'un foyer dans une société débarrassée de la propriété privée ne sera lié que par un véritable amour et Katō Midori souligne que les femmes recherchent de la nouveauté et un sentiment de plénitude (kibun no jüjitsu 気分の充実) :

" (...) les femmes se sont lassées de leur vie ordinaire et calme, comme endormie. Elles sont donc toujours attirées par quelque chose. Je veux parler de la plénitude des sentiments. Elles ne peuvent se satisfaire en fin de compte de l'amour des hommes, qui ne connaît que le désir charnel. Nous voulons davantage de satisfactions qui allient le spirituel et le physique. Nous voudrions pouvoir nous observer nousmêmes de l'extérieur, et nous ne regardons plus les hommes avec respect comme avant. »(Horiba 1991 : 104).

Il y a donc une opposition entre les sentiments rendus possibles dans une société déterminée par les hommes et ceux que les femmes veulent 
pouvoir exprimer. Raichō insiste davantage sur l'épanouissement créatif des femmes, Itō parle de l'épanouissement des pionnières. Il faut remarquer que l'accent mis sur les sentiments et la nécessité de l'amour dans le mariage est rendu quasiment inévitable par la conscience que les femmes ont d'être instrumentalisées dans le mariage pour servir des intérêts économiques. L'explication de Fukuda Hideko sur l'avenir du mariage est à ce propos très éclairante. Pour changer le mariage, il faut se débarrasser de la propriété privée, car ainsi il ne pourra plus y avoir de mariage par intérêt.

Un autre point essentiel qui rapproche ces textes est l'idée que la femme est d'abord un être humain. Raichō et Fukuda ne se retrouvent-elles pas sur la même idée quand l'une dit qu'elle ne s'est jamais pensée comme femme et quand l'autre affirme que la femme ne sera pas libre tant que l'homme ne le sera pas? Quand Iwano Kiyo démontre que la discrimination entre les sexes est idiote puisque la détermination du sexe suit la formation de l'être vivant et que par ailleurs certaines personnes changent de sexe ou n'ont pas un sexe clairement défini, n'est-ce pas une manière de dire un peu la même chose : l'être humain préexiste au genre, et ce dernier ne doit pas déterminer la vie des humains ? Katō Midori affirme, elle, que la femme doit s'éveiller à sa nature d'être humain et quitter sa conscience de femme, ce qui est une manière de faire le même chemin que Raichō dans l'autre sens, même si elle souligne que l'homme et la femme vivent dans des mondes différents, ce qui peut être compris comme une contradiction et reflète un aspect du débat sur les femmes encore vivant de nos jours.

\section{Conclusion}

Les femmes de la revue Seitō ont parfois été critiquées par des féministes qui les considéraient comme des bourgeoises exprimant des préoccupations subjectives. Ainsi, la socialiste Yamakawa Kikue 山川菊栄 (1890-1980) qui, sous le nom d'Aoyama Kikue 青山菊栄, débattit dans la revue avec Itō Noe, Hiratsuka Raichō et Yosano Akiko 与謝野晶子, insiste sur leur romantisme, leur vue partielle des problèmes et leur subjectivité (Yamakawa 1978 : 193195). L'accent mis sur l'expression de soi, sur la créativité et sur l'épanouissement des sentiments leur est reproché, car cela dénoterait un manque de sens politique et de vision sociale de la place de la femme. Et il est vrai que l'histoire de l'association regorge d'épisodes de relations personnelles 
houleuses, d'histoires d'amour compliquées et parfois rocambolesques. Mais en même temps, une question se pose : pouvait-il émerger au Japon à cette époque un mouvement de femmes qui soit purement ancré dans le politique, quand on considère que les femmes étaient totalement exclues par la loi de la sphère politique ? Est-ce vraiment un hasard si c'est plutôt la fibre artistique, littéraire, des femmes qui s'est exprimée ainsi au grand jour à l'ère Taishō ? Et il reste que c'est bien grâce à Seitō que Yamakawa Kikue elle-même s'exprimera pour la première fois, même si elle ne sera jamais membre du groupe. C'est bien aussi dans Seitō que Fukuda Hideko publia sa vision de la femme nouvelle. Le fait d'être une revue littéraire donna à Seitō une liberté de choix des thèmes et une liberté de ton singulières. Les thèmes abordés, qui vont jusqu'à la prostitution et à la sexualité, sont d'une grande audace pour l'époque. Yamakawa Kikue reconnaît d'ailleurs que la sincérité des poèmes de Yosano Akiko a vraiment touché les lecteurs et contribué à affaiblir la morale conservatrice (Yamakawa 1978 : 194).

Par ailleurs, sur le thème de la femme nouvelle, la réflexion menée dans Seitō ne manque pas de profondeur et révèle bien la rupture qui existe entre les propos de l'ère Meiji sur les femmes, majoritairement tenus par des hommes, et l'ère Taishō. La lecture approfondie de ce débat entre femmes permet de mesurer le chemin parcouru par le Japon. Ce sont bien des femmes qui parlent en leur nom propre cette fois, et leurs préoccupations nous semblent plus proches de notre époque que de celle de Fukuzawa Yukichi. La plupart des questions qu'elles abordent n'ont toujours pas été réglées et continuent à faire débat, ce qui est pour le moins la preuve du caractère à la fois novateur pour l'époque et en même temps actuel de leur questionnement.

Cent ans après la fondation de Seitō, force est de constater que ce débat sur la femme nouvelle et sur l'égalité des sexes garde toute son actualité. Au niveau mondial, des situations pires que celles dénoncées en 1913 par les femmes japonaises existent bel et bien. La violence des propos d'Itō Noe n'est malheureusement rien comparée à la violence faite aux femmes partout dans le monde. Les larmes et les souffrances dont nous parlent les femmes de Seitō sont sans doute les mêmes que celles d'une Taslima Nasreen pour ne parler que d'une des plus célèbres.

À l'échelle du Japon, l'analyse du choix du célibat proposée par Katō Midori résonne familièrement à nos oreilles contemporaines. À une époque 
où beaucoup de femmes choisissent de vivre seules, les médias japonais regorgent aussi d'articles sur le konkatsu 婚活, la course au conjoint, notamment pour les femmes qui ne peuvent envisager d'assurer à long terme leur indépendance économique dans un contexte de crise et de précarisation des emplois. Par ailleurs, le débat sur les femmes et leur place dans la vie économique par exemple se trouve souvent piloté par la conjoncture économique, et l'analyse de Katō Midori sur l'émergence cyclique de femmes nouvelles et les éléments qui en favorisent l'apparition se trouve donc régulièrement vérifiée. Des sociologues (Yamada 2010 : 34) constatent la difficulté actuelle des hommes japonais à garantir l'aisance matérielle d'une famille en raison de la grande précarité des emplois. Ils pointent du doigt la baisse du nombre des mariages qui s'ensuit, et le lien avec la baisse de la natalité. On ne peut que reconnaître, comme Katō Midori, que dans ce contexte, le rôle économique des femmes cesse d'être une simple force d'appoint, même si elles restent encore les premières victimes de la précarisation.

\section{Bibliographie}

\section{DODANE Claire 2000}

Yosano Akiko, poète de la passion et figure de proue du féminisme japonais, Paris, Publications orientalistes de France.

FUKUZAWA Yukichi 福澤諭吉 2003

Fukuzawa Yukichi chosakushū 福澤諭吉 著作集 (Recueil de textes de Fukuzawa Yukichi), t. X, Tokyo, Keiō gijuku daigaku shuppankai 慶應義塾大学出版会.

\section{HIRATSUKA Raichō 平塚らいてう 1992}

Genshi, josei wa taiyō de atta 元始、女性は 太陽であった (À l'origine, la femme était le soleil), Tokyo, Ōtsuki shoten 大月書店.
HORIBA Kiyoko 堀場清子 1988

Seitō no jidai 『青靼』の時代 (L'époque de Seitō), Tokyo, Iwanami shoten 岩波書店.

HORIBA Kiyoko (dir.) 1991

Seitō josei kaihō ronshū 『青靼』女性解

放論集 (Seitō recueil de textes sur la libération des femmes), Tokyo, Iwanami shoten 岩波書店.

IDE Fumiko 井手文子

\& ESASHI Akiko 江刺昭子 1977

Taishō demokurashī to josei

大正デモクラシーと女性 (La démocratie

Taishō et les femmes), Tokyo,

Gōdō shuppan 合同出版. 
KANEKO Sachiko 金子幸子 1999

Kindai Nihon joseiron no keifu 近代日本 女性論の系譜 (Généalogie des discours sur les femmes japonaises modernes), Tokyo, Fuji shuppan 不二出版.

\section{LÉVY Christine 2011}

« Hiratsuka Raichō et le refus du modèle familial moderne », in GALAN Christian et LOZERAND Emmanuel (dir.), La famille japonaise moderne 1868-1912. Discours et débats, Arles, Picquier, pp. 445-460.

\section{MACKIE Vera 2003}

Feminism in Modern Japan,

Cambridge, Cambridge University Press.

NARITA Ryūichi 成田隆一 2007

Taishō demokurashī 大正デモクラシー

(La démocratie de Taishō), Tokyo,

Iwanami shoten 岩波書店.

Seitōsha 青鞜社 1911-1916

Seitō. Fukkoku-ban 青鞜復刻版

(Seitō. Fac-similé), Tokyo, Fuji shuppan

不二出版, 1983.

Shin feminizumu hihyō no kai

新・フェミニズム批評の会

(Association pour une nouvelle critique féministe) (éd.) 1998

Seitō o yomu『青靼』を読む (Lire Seitō), Tokyo, Gakugei shorin 學藝書林.

SOTOZAKI Mitsuhiro 外崎光弘 1986

Nihon fujinronshi 日本婦人論史

(Histoire des discours sur les

Japonaises), vol. 1 et 2, Tokyo,

Domesu shuppan ドメス出版.
YAMADA Masahiro 山田昌弘 2010 Konkatsu genshō no shakaigaku 婚 活現象の社会学 (Le phénomène de konkatsu, une sociologie de la course au conjoint]), Tokyo, Tōyō keizai 東洋経済.

YAMAKAWA Kikue 山川菊栄 1978 Nijusseiki o ayumu 二十世紀をあゆむ (Parcourir le vingtième siècle), Tokyo, Daiwa shobō 大和書房. 